\title{
Kernos
}

Revue internationale et pluridisciplinaire de religion grecque antique

$27 \mid 2014$

Varia

\section{Les Grecs et le cru}

Pratiques alimentaires, pratiques rituelles et représentations dionysiaques

\section{Carine Van Liefferinge}

\section{(2) OpenEdition}

\section{Journals}

Édition électronique

URL : http://journals.openedition.org/kernos/2272

DOI : 10.4000/kernos.2272

ISSN : 2034-7871

\section{Éditeur}

Centre international d'étude de la religion grecque antique

\section{Édition imprimée}

Date de publication : 1 novembre 2014

Pagination : 75-97

ISBN : 978-2-87562-055-2

ISSN : 0776-3824

\section{Référence électronique}

Carine Van Liefferinge, « Les Grecs et le cru », Kernos [En ligne], 27 | 2014, mis en ligne le 01 octobre 2016, consulté le 20 avril 2019. URL : http://journals.openedition.org/kernos/2272 ; DOI : 10.4000/ kernos. 2272

Ce document a été généré automatiquement le 20 avril 2019. 


\title{
Les Grecs et le cru
}

\author{
Pratiques alimentaires, pratiques rituelles et représentations \\ dionysiaques
}

Carine Van Liefferinge

1 Oribasie, sparagmos et ômophagie sont traditionnellement considérés comme les trois phases successives du rituel dionysiaque dont la violence bestiale, l'étrangeté ou même simplement la performance physique ont dérangé et conduit, soit à concevoir ces pratiques comme marginales, "a-civiques » mais réelles, soit à émettre des doutes sur leur réalité. Dans une intéressante étude, Monique Halm-Tisserant souligne en effet, à propos de l'ômophagie, le laconisme des sources littéraires et figurées' ${ }^{1}$. Aussi s'intéresse$\mathrm{t}$-elle plus particulièrement au sparagmos, celui-ci devenant selon elle le point d'orgue du rituel. Pratiqué à mains nues, il comporte une phase de section en deux de la victime en position horizontale, suivie du démembrement de l'animal. Assez étonnamment, la réalité de ce rituel, qui requiert, de la part des bacchantes, une force peu commune, n'est pas ici remise en cause. L'argumentation de l'auteur, fondée essentiellement sur l'iconographie, l'amène au contraire à parler d'un rite dont la réalité est affirmée par la constance des thèmes dans la représentation figurée, qui vient s'ajouter à l'usage d'une terminologie précise dans les textes. Quoi qu'il en soit, on doit à cette recherche d'avoir relancé le débat sur la question de l'ômophagie. M. Halm-Tisserant montre en effet de façon convaincante qu'une fois le démembrement effectué, il n'y a pas consommation des parts de viande crue, mais bien plutôt un lancer rituel de celles-ci, lancer à la symbolique fécondante puisque son objectif serait d'apporter à la terre l'énergie de la chair vivante. Et de conclure: "Tenir le sparagmos dionysiaque pour un rituel d'ensemencement de la terre a pour contrepartie d'ôter à l'ômophagie sa raison d'être " $»^{2}$. Ainsi, par le biais d'une étude sur le sparagmos, en arrive-t-elle à remettre en cause la réalité de l'ômophagie. Nous avons voulu reprendre la question frontalement et, après d'autres, sommes partie à la recherche des traces d'une pratique ômophagique dionysiaque effective. Aucune pièce nouvelle n'est à verser à un dossier déjà maintes fois commenté et interprété. Toutefois, par une étude plus large du « manger cru » dans la culture et l'imaginaire des Grecs et par la mise en regard de leur pratique alimentaire et de certaines pratiques cultuelles en 
l'honneur de Dionysos, il nous a semblé possible de cerner, dans des témoignages d'époque, de lieu et de nature différents, des représentations du dieu partagées.

\section{Le « manger cru » dans la pratique alimentaire}

2 Personne ne contesterait qu'étudier le mode d'alimentation d'un individu ou d'un peuple puisse révéler les valeurs morales, sociales ou politiques auxquelles il est attaché ${ }^{3}$. Aussi, avant d'en arriver à l'ômophagie rituelle, avons-nous replacé le « manger cru » dans les habitudes alimentaires des Grecs : les Grecs mangeaient-ils cru? Que mangeaient-ils cru? À quels êtres réels ou fictifs le « manger cru » est-il assigné ? Quels débats ont pu susciter

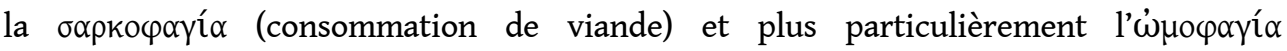
(consommation de viande crue), ce dernier terme n'étant manifestement pas réservé à un cadre rituel?

3 L'adjectif $\omega ̉ \mu o ́ \varsigma$ peut évoquer le cru par opposition au cuit dans le cas d'une nourriture quelle qu'elle soit (légume ou viande), le pas mûr dans le cas d'un fruit vert qui n'est pas encore cuit par le soleil ${ }^{4}$. Divers témoignages attestent que les Grecs absorbaient les aliments crus, bouillis et rôtis/grillés. Au livre II du traité hippocratique du Régime, on trouve un véritable catalogue d'aliments, avec leurs qualités et leurs préparations, considéré par R. Joly comme le plus complet du temps ${ }^{5}$; d'une façon générale, l'auteur du

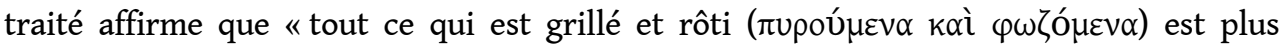
resserrant que ce qui est cru parce que, sous l'effet du feu, l'humide, le jus et le gras sont enlevés $»^{6}$. Ailleurs encore, parlant des fruits du chêne, il avance que les glands sont

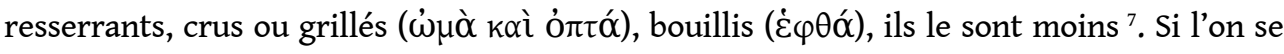
fonde sur ce traité, il semble que ce sont les légumes et les fruits qui sont consommés crus ; à notre connaissance, aucun extrait ne fait mention d'absorption de viande crue. Un passage de Plutarque semble confirmer que le rôti et le bouilli sont les deux modes de préparation des mets puisque, dans son traité sur la colère, il fustige ceux qui se mettent en colère pour la raison futile que les mets sont trop rôtis (ómtolovv) ou trop bouillis $(\dot{\varepsilon} \varphi \theta 0 ı c)^{8}$. Plutarque encore, dans les Questions romaines, s'interroge sur les interdits alimentaires du flamen Dialis ${ }^{9}$ : pourquoi le prêtre de Zeus s'abstient-il de toucher à la

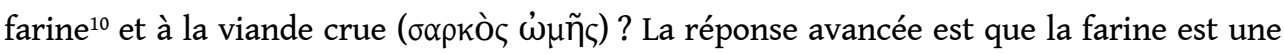

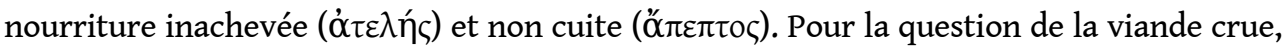

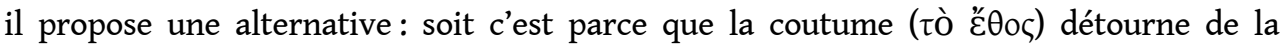
consommation de viande crue ( $\dot{\omega} \mu \circ \varphi \alpha \gamma i ́ \alpha)$, soit c'est parce que la viande fraîche et crue

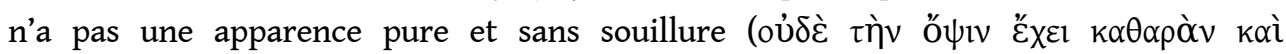
$\dot{\alpha} \mu$ í $\alpha v \tau$ tov). On comprend également que la viande crue, comme la farine, se trouve dans un état intermédiaire entre le vivant et la nourriture : « Elle n'est ni un être vivant ni déjà une nourriture cuite; car la cuisson et le rôtissage, étant une altération et un changement, changent la forme $»^{11}$. Dans une analyse fondée sur le couple nature/ culture, Jean-Pierre Vernant relève cette notion d'impureté qui rend intouchable la viande de l'animal sacrifié qui n'a pas encore reçu, par cuisson, sa pleine forme de mets humains ${ }^{12}$.

Deux notions relevées ci-dessus se retrouvent dans un passagedu discours de Julien Contre les cyniques ignorants, celle d'habitude alimentaire et celle d'impureté de la viande crue. Dans ce discours, il s'en prend aux philosophes cyniques de son temps qui discréditaient

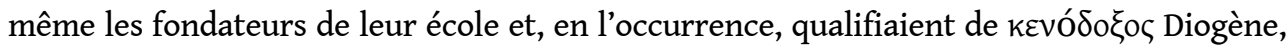


mort suite à la consommation de la chair crue d'un poulpe. On comprend plus loin le sens de cet adjectif: Diogène, qui visait l'apathie ( $\dot{\alpha} \pi \alpha \theta_{\varepsilon \varepsilon 1 \alpha)}$ propre aux dieux, était pris de

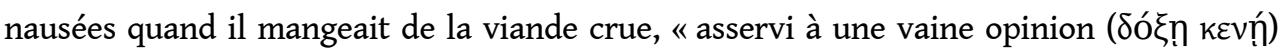
plus qu'à la raison ». Aussi décida-t-il de se libérer de ce dégoût qu'il considérait comme une faiblesse en s'imposant la consommation de la chair crue d'un poulpe. Julien entreprend alors une digression sur la question de la consommation de viande

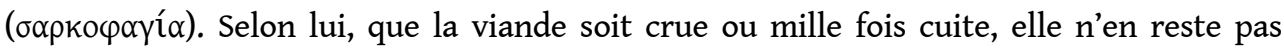
moins de la viande ; la cuisson n'est qu'une question d'usage et d'habitude, et considérer la chair cuite comme pure et mangeable relève de la $\delta$ ó $\alpha$. Julien se demande donc pourquoi ne pas consommer la viande tout simplement ( $\dot{\alpha} \pi \lambda \tilde{\omega} \varsigma$, opposé à $\sigma \alpha \rho \kappa \tilde{\omega} v$ $\dot{\varepsilon} \psi \eta \mu \varepsilon ́ v \omega v)$, comme le font d'ailleurs ceux qui habitent près de la mer « qui avalent, sans même les faire chauffer, des oursins et des huîtres ${ }^{13}$. Cette absorption d'un poulpe cru par Diogène ne relève certainement pas de l'anecdote, mais s'inscrit dans l'intention plus large, de la part des philosophes cyniques, de se démarquer du modèle sacrificiel et donc alimentaire de la cité, ce qui signifie que le manger cru ne semble pas faire partie des habitudes alimentaires des Grecs et, quoi qu'en dise Julien, passe même à leurs yeux pour une pratique sauvage et barbare, propre au monde animal, comme nous allons le voir.

Revenons à l'adjectif $\omega$ uóc. Il convient encore de signaler qu'il peut être utilisé dans un sens moral, ainsi que son contraire : l'adjectif $\pi \dot{\varepsilon} \pi \omega v$, appliqué au fruit mûr, peut signifier

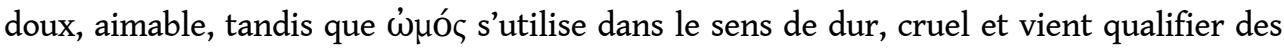
sentiments violents tels que la colère ${ }^{14}$. Il est d'ailleurs remarquable que, sous le coup de la colère, les héros épiques - et les dieux - en arrivent à souhaiter commettre des actes cruels et que cette cruauté s'illustre précisément par l'acte de manger cru. Ainsi, pour guérir le courroux d'Héra contre les Troyens, il lui faudrait, dit Zeus dans l'assemblée des dieux du chant IV de l'Iliade, dévorer crus Priam, ses fils et tous les Troyens ${ }^{15}$. Autre exemple : Achille voudrait que sa colère le pousse à dévorer le corps d'Hector cru, après

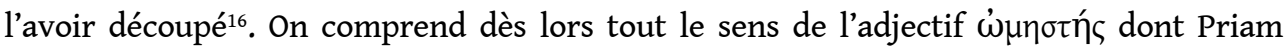
qualifie Achille avant de se rendre chez lui réclamer le corps d'Hector ${ }^{17}$, adjectif généralement traduit par «cruel » mais qui veut bien dire " dévoreur de chair crue » si on met en parallèle les deux extraits cités ici ${ }^{18}$. Manger de la viande humaine crue apparaît comme un acte d'une cruauté extrême, seul capable d'apaiser une colère extrême. C'est aussi l'acte qui figure l'anéantissement de l'ennemi : dans l'Anabase par exemple, Xénophon exhorte ses troupes à manger l'ennemi tout cru si possible ${ }^{19}$.

De ce qui précède on soupçonne que si le manger cru peut s'appliquer à d'autres aliments que la chair animale ou humaine, comme à des légumes ou des fruits, l'être qualifié

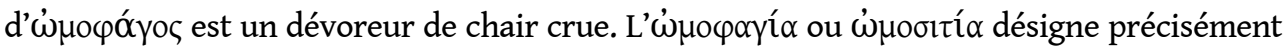
l'absorption de viande crue, acte qui, aux yeux des Grecs, est le propre de certains animaux ou d'êtres primitifs, barbares, sauvages ou monstrueux.

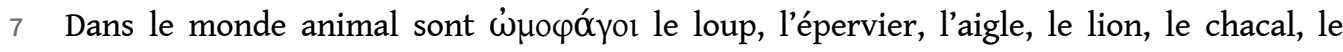
faucon ${ }^{20}$. Sont $\omega \dot{\omega}$ $\sigma \tau \alpha$ le les oiseaux ou les chiens qui dévorent les cadavres auxquels on ne rend pas les hommages funèbres ${ }^{21}$. Sont encore mangeurs de chair crue certains peuples sauvages (ou présentés comme tels, en tout cas) comme les Eurytanes, groupement étolien, pointés par Thucydide ${ }^{22}$ ou les Tarikhanes, peuple sorti de l'imagination de Lucien $^{23}$, gens aux yeux d'anguille, à la figure de homard, belliqueux, audacieux et

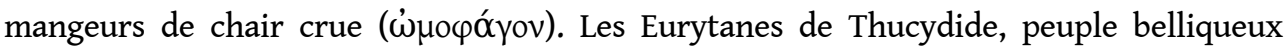

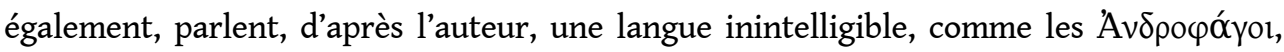
peuple anthropophage voisin des Scythes, peuple sauvage et sans loi, dont parle Hérodote 
${ }^{24}$, ce qui situe l'ômophagie à la limite du cannibalisme. Enfin, sont ômophages toute une série de monstres de la mythologie, caractérisés par leur appartenance partielle au monde animal ou par leur sauvagerie : la Sphinx est $\omega \mu$ ó griffes sont avides de viande vivante ${ }^{26}$. Le chien des Enfers, Cerbère, est $\omega \mu \eta \eta \tau t \eta ́ \varsigma^{27}$,

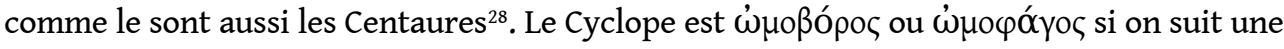
autre leçon manuscrite ${ }^{29}$. Et pour Lucien encore, celui à qui on doit la paternité de toutes sortes d'histoires monstrueuses est l'Ulysse d'Homère qui raconte « la férocité de certains

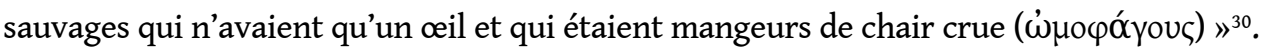

8 Il se confirme ainsi que manger de la viande crue ne faisait pas partie des habitudes alimentaires traditionnelles des Grecs, mais qu'au contraire cet usage apparaît comme une contre-valeur. L'analyse structurale des mythes comme celui de Prométhée a depuis longtemps mis en lumière les significations que les Grecs ont données à la cuisson par le feu. Nous rappellerons simplement ici qu'en enlevant le feu aux hommes, Zeus rabaisse l'homme au rang des bêtes, l'obligeant à manger cru comme celles-ci. Le sacrifice traditionnel et les pratiques alimentaires qui en découlent sont sous-tendus par les relations entre les trois termes dieux - hommes - animaux. La différence entre l'homme et l'animal, qui ont comme point commun de devoir échapper à la faim pour vivre, se situe au plan alimentaire : "L'homme n'est pas un animal mangeur de viande crue », dit Porphyre $^{31}$. Parmi les animaux, ceux qui sont considérés comme les moins sauvages sont les herbivores ${ }^{32}$. Et même parmi les plantes, la plante véritablement sauvage n'admet aucune coction, qu'il s'agisse de celle du soleil ou de celle de la préparation culinaire ${ }^{33}$. Cette association avérée du cru et du sauvage a immanquablement orienté l'interprétation structuraliste de l'ômophagie, selon laquelle, dans la religion dionysiaque, la dévoration des chairs crues d'un animal sauvage qui n'a pas été mené à l'autel ni cuisiné rituellement signifie l'effondrement de la barrière entre l'humanité et la bestialité, et l'accès à une sauvagerie considérée comme étant propice au contact direct avec le divin ${ }^{34}$.

\section{Le « manger cru » dans la pratique rituelle dionysiaque}

9 L'indéniable relation entre la pratique alimentaire et le sacrifice amène à envisager le contexte religieux dans lequel s'insérerait le manger cru. Car, si les Grecs évacuaient le cru de leur alimentation, en allait-il de même dans la pratique rituelle? En d'autres termes, quand il est question d'ômophagie rituelle, faut-il l'entendre au sens étymologique du terme?

\section{L'ômophagie entre mythe et réalité}

10 Depuis l'étude fondamentale d'Albert Henrichs sur le ménadisme, il est globalement acquis que la pratique du « manger cru » dionysiaque relève du mythe ou de la métaphore 35. Ainsi, le témoignage le plus célèbre, celui d'Euripide dans les Bacchantes, qui a souvent été lu comme l'évocation mythique de pratiques liées à l'un ou l'autre rituel dionysiaque, a été réévalué. En effet, ce que nous y relevons, ce n'est pas tant le déchirement de Penthée par sa propre mère Agavé, possédée par le dieu, déchirement qui n'est d'ailleurs pas suivi de la consommation de ses chairs crues (mais seulement d'une invitation à un festin ${ }^{36}$ ), que les allusions au culte dispersées dans le chœur des Bacchantes lydiennes qui accompagnent le dieu. Une seule mention d'une pratique ômophage s'inscrit dans des 
vers où le chœur exprime sa joie de retrouver une nature libre et sauvage : «Il est doux, sur les montagnes, après la course des thiases, de tomber sur le sol, vêtu de la nébride sacrée, de chasser le bouc afin de se repaître de son sang, d'en manger la viande crue

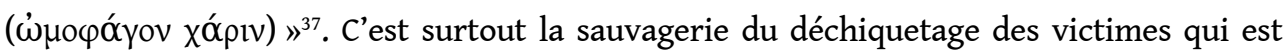
décrite : « Elles assaillent à mains nues nos génisses qui paissaient dans l'herbe ; tu aurais pu en voir une déchirer en deux, de ses mains, une jeune vache aux mamelles gonflées, mugissante, et les autres mettre des génisses en lambeaux. Tu aurais pu voir, lancés en tout sens, ici des côtes, là un sabot fourchu, suspendus, dégouttants de sang, aux sapins. Des taureaux furieux, aux cornes menaçantes, sont jetés à terre, puis emmenés par mille mains de jeunes femmes. Les chairs sont dépecées plus vite que tes paupières ne couvrent tes royales prunelles $»^{38}$.

11 Dans ces vers, comme l'a relevé M. Halm-Tisserant, pas d'ômophagie consécutive au sparagmos. Sur la folie suscitée chez les femmes par Dionysos, on peut lire encore chez le pseudo-Apollodore : « Après avoir ainsi montré aux Thébains qu'il était un dieu, Dionysos se rendit à Argos et là, comme on refusait à nouveau de lui rendre un culte, il frappa les femmes de folie. Elles emportaient dans les montagnes les enfants encore à la mamelle et en dévoraient la chair ${ }^{39}$. Le mythographe semble avoir réuni les éléments que l'on trouve chez le poète tragique, rappelant, de façon synthétique, les difficultés que Dionysos rencontre tout au long de sa geste à faire reconnaître sa divinitée ${ }^{40}$, ce qu'Euripide avait également montré de façon insistante : nouveau dieu ${ }^{41}$, dieu étranger ${ }^{42}$, Dionysos voit son statut de dieu contesté, ainsi que sa naissance de la cuisse de Zeus ${ }^{43}$. Les actes horribles auxquels il pousse les bacchantes sont là pour montrer sa puissance. Et le sparagmos de Penthée par sa propre mère est la punition qu'il inflige au roi de Thèbes pour avoir refusé d'admettre sa divinité et pour avoir cru pouvoir berner le dieu par son intelligence humaine.

12 Dans la perspective de la tragédie, c'est bien plus l'hybris de Penthée que la cruauté de Dionysos qui est présentée. La faute de Penthée est un excès de confiance dans le pouvoir de l'intelligence humaine ${ }^{44}$. Dionysos n'est d'ailleurs pas le seul dieu mis en scène par la tragédie à pousser aux pires actes les hommes coupables d'actes d'hybris, le plus bel exemple étant le sacrifice d'Iphigénie commis par Agamemnon sur l'ordre d'Artémis. Ainsi, pas plus que le sacrifice d'Iphigénie n'autorise à déclarer effective la pratique du sacrifice humain, les agissements des bacchantes n'autorisent à penser que les femmes grecques quittaient réellement la sphère de la maison pour aller sur la montagne déchiqueter des animaux sauvages et en consommer la chair encore palpitante. D'autres sources, postérieures, reprennent de façon évidente la description fournie par Euripide et il est plus que probable que celle-ci a servi de modèle. Ainsi lit-on notamment chez Lucien : «Les femmes s'emparent de troupeaux, les déchiquettent car certaines d'entre elles sont ômophages $»^{45}$.

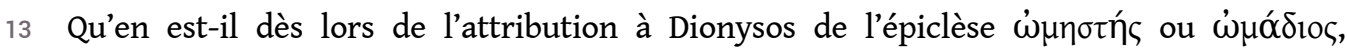
« mangeur de chair crue »? Comme épithète, nous l'avons déjà rencontrée pour qualifier certains animaux ou encore Achille ${ }^{46}$; comme épiclèse, elle semble n'avoir été attribuée qu'à ce seul dieu ${ }^{47}$. Elle lui est appliquée, aux côtés de nombreuses autres et sans mise en contexte, dans un Hymne orphique ${ }^{48}$, usage étonnant, comme le souligne A.-Fr. Morand, dès lors que le mode de vie orphique invite au végétarisme et condamne le sacrifice sanglant ${ }^{49}$. Elle lui est également attribuée par Plutarque et par Porphyre dans des contextes plus

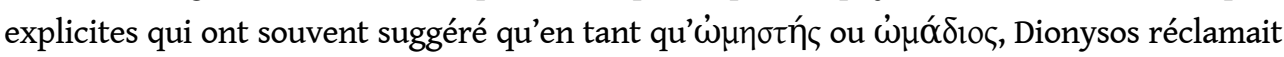
des sacrifices humains. Dans la Vie de Thémistocle, Plutarque, se fondant sur le témoignage 
du philosophe Phainias d'Érésos, raconte qu'à Salamine, suite à différents présages interprétés par un devin, Thémistocle immole, sous la pression de la foule en délire, trois magnifiques prisonniers de guerre perses à Dionysos Ômèstès, afin d'assurer la victoire aux Grecs. Pierre Bonnechere met en doute l'historicité de ce sacrifice, avançant des arguments pour ou contre qu'il serait trop long de reprendre ici. Retenons seulement de son argumentation, avec les limites que ce résumé lui impose, que le nom de Dionysos Ômèstès n'est nulle part attesté en Attique et que son culte est limité à trois îles de la mer Égée, Chios, Ténédos et Lesbos d'où est originaire Phainias ${ }^{50}$.

Les mentions de Chios et de Ténédos proviennent des témoignages suivants. On trouve chez Eusèbe de Césarée ${ }^{51}$ cette citation de Porphyre qui lui-même cite Euelpis de Carystos - c'est d'ailleurs le seul passage conservé de cet historien dont on ne trouve aucune mention ailleurs ${ }^{52}-$, citation brève et peu détaillée : «À Chios également, on sacrifiait à Dionysos Ômadios un homme que l'on déchiquetait, ainsi qu'à Ténédos comme le rapporte Euelpis de Carystos ${ }^{53}$. Selon P. Bonnechere, si ce témoignage, ainsi qu'un autre, aussi peu explicite et cité par Clément, lui-même cité par Eusèbe, faisant état à Lesbos de sacrifice humain à Dionysos, ne peut suffire à nier l'existence de sacrifices humains dionysiaques, on ne peut non plus y trouver une preuve sûre de leur réelle existence: Porphyre, Eusèbe et Clément en ont parlé dans des écrits engagés, l'un, contre le sacrifice sanglant, les autres, contre le paganisme, religion de démons malfaisants. Selon lui toujours, ces sacrifices faisaient probablement partie de listes qu'on se plaisait à répéter. Il conclut en disant que les sacrifices humains qui apparaissent dans les fêtes dionysiaques sont tous mythiques et remplissent la fonction « de dramatiser, de ritualiser à l'extrême une brisure radicale dans le cours de la vie des hommes, des femmes, des cités ${ }^{54}$. Cette antinomie entre les rites dionysiaques et ceux préconisés par l'ordre social avait déjà été soulignée par M. Detienne dans ses travaux sur Dionysos.

Mais revenons-en à la question de l'ômophagie. Nous aurons pu constater que dans les témoignages de Plutarque et de Porphyre, qui font l'un et l'autre état de sacrifice humain dont la réalité historique est douteuse voire contestable, il n'est en tout cas pas question de dévorer crue la victime sacrifiée, acte qui relèverait ici de l'anthropophagie ${ }^{55}$. Même si l'argument a silentio peut être difficile à manier, on peut supposer que, si la victime humaine sacrifiée à Dionysos avait été consommée crue, Porphyre n'aurait manqué de le mentionner dans un traité sur le végétarisme où il énumère les horreurs que sont capables de commettre les adeptes du sacrifice sanglant. En outre, il importe de souligner que c'est Dionysos qui, recevant ce type de sacrifice, est métaphoriquement appelé « mangeur de chair crue ». L'épiclèse mérite qu'on s'y arrête. En effet, vu le petit nombre d'occurrences de ces épiclèses, leur contexte vague ou géographiquement limité, la prudence impose de ne pas généraliser à l'ensemble du culte dionysiaque ce qui pourrait n'être qu'une particularité locale. Quant à l'aspect cultuel auquel l'épiclèse renverrait, il semble que c'est le dieu qui est «ômophage » plutôt que ses adeptes. Nous verrons plus loin que, dans un certain sens, cette hypothèse, déjà avancée par Albert Henrichs ${ }^{56}$, n'est pas à exclure. Enfin, pour en revenir à la question de la réalité du rituel évoqué par Porphyre, ajoutons qu'elle a sans doute été affirmée parce qu'une relation a parfois été établie entre sacrifice humain et ômophagie. Ainsi, Étienne Coche de la Ferté interprète la tragédie d'Euripide comme une affabulation littéraire d'un rite religieux très ancien qui consistait à mettre à mort en le déchirant un représentant du dieu et de s'assimiler la puissance de celui-ci par le rite de l'ômophagie. Il en conclut que l'ômophagie avait pour point de départ le sacrifice humain et que, celui-ci évoluant vers le sacrifice animal, les 
animaux qui furent mangés crus étaient des victimes de substitution ${ }^{57}$. Il a été montré avec beaucoup de pertinence que l'on peut facilement renverser cette théorie et défendre l'idée que «la réelle ômophagie animale, qui paraissait barbare et ancestrale, aurait suscité dans le mythe, par essence exagérateur, l'idée d'antiques immolations d'êtres humains à Dionysos $»^{58}$. Il n'en reste pas moins que cette interprétation continue de considérer que l'ômophagie animale pourrait être « réelle». Or, le texte de Porphyre est loin de le suggérer.

\section{L'ômophagie : un rituel exclusivement dionysiaque ?}

est convenu d'affirmer que la pratique du manger cru s'inscrit exclusivement dans la sphère dionysiaque. Tous les témoignages ne vont cependant pas aussi nettement dans ce sens. Dans le De defectu oraculorum de Plutarque, à la question de la cause de la décadence de l'oracle, Cléombrote, un des intervenants, avance une théorie démonologique, attribuant notamment à des démons, et non à des dieux, toute une série de faits parfois monstrueux rapportés par les récits mythiques, ainsi que toutes sortes de pratiques rituelles : « En ce qui concerne les cérémonies des mystères, dans lesquelles il est possible de saisir les indices les plus grands et les plus clairs de la vérité au sujet des démons, "que ma bouche garde le silence", comme dit Hérodote. Mais en ce qui concerne des fêtes et des sacrifices, comme des jours néfastes et lugubres, au cours desquels on pratique des ômophagies et des déchirements, on s'adonne à des jeûnes et on se frappe la poitrine, et qu'en maint endroit les sanctuaires renvoient des paroles obscènes, "des délires et des clameurs émanant de ceux qui, la nuque ployée, sont agités”, je dirais qu'ils ne sont accomplis pour aucun dieu, mais en vue de détourner de mauvais démons, en les apaisant et les calmant $~^{59}$. Partons du constat que l'auteur de ces propos distingue d'une part les

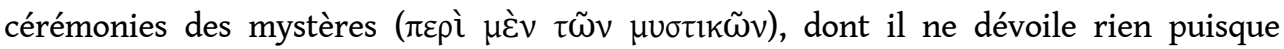

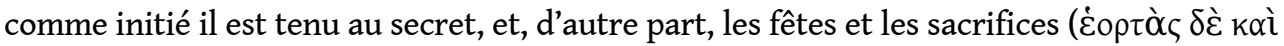
Avoíac) où se produisent l'ômophagie et d'autres pratiques condamnables. On aura noté également que les mystères révèlent la vérité au sujet des démons, tandis que ces pratiques sont destinées à de mauvais démons.

17 Avant de revenir à ces démons, envisageons une première question : est-ce bien Dionysos qui est évoqué dans ce passage? Une citation de Pindare ne laisse aucun doute à ce sujet : il s'agit d'un vers, extrait d'un dithyrambre, dans lequel le poète montre la nature entière et les autres dieux honorant Dionysos ${ }^{60}$. Ce vers a été introduit par Plutarque dans l'évocation des pratiques rituelles que manifestement il condamne. Cette lecture impliquerait-elle que Dionysos est un de ces "mauvais démons" auxquels elles s'adressent ? Cela paraît étonnant quand on sait par Plutarque lui-même qu'il était initié aux mystères dionysiaques ${ }^{61}$. En fait, c'est toute l'ambiguïté du dieu qui se dévoile une nouvelle fois ici. Une figure double, l'une, démonique, celle du dieu « caché » ${ }^{62}$ qui doit affirmer sa divinité, l'autre divine, lorsqu'enfin reconnu comme dieu, Dionysos accède à l'olympe. Les rituels ômophagiques (qu'il reste encore à définir) seraient adressés à la première. Précisons de quel Dionysos il pourrait s'agir ici, en intégrant les données de la tradition orphique, notamment celle des Hymnes contemporains du texte de Plutarque.

En effet, Jean Rudhardt a souligné les points de convergence entre les deux traditions qui concernent la naissance de Dionysos, celle qui fait de lui le fils de Sémélé et celle qui fait de lui le fils de Perséphone. Il a montré de façon convaincante que les Hymnes orphiques «ne juxtaposent pas inconsidérément les données de deux traditions distinctes; ils en 
proposent une synthèse, en enseignant sans doute possible que Dionysos a eu deux mères successives. Une donnée fondamentale du mythe orphique peut seule expliquer ce paradoxe : il raconte le meurtre commis par les Titans sur la personne de Dionysos. Il faut que l'enfant de l'une des deux mères disparaisse, pour qu'il ait à naître une seconde fois ». Dionysos est l'enfant de Perséphone. Lorsqu'il fut démembré par les Titans, son cœur a été sauvé par Athéna et confié à Zeus. Celui-ci trouve en Sémélé une seconde mère pour porter l'enfant ${ }^{63}$.

Si l'on suit cette interprétation, dans la temporalité mythique, le « premier » Dionysos est l'enfant de Perséphone, à savoir celui de la tradition orphique, le « second » est le fils de Sémélé. En tant que fils de Zeus et de Perséphone, il est divin; en tant que fils de la mortelle Sémélé, il a des éléments mortels qu'il faut éliminer: ce qui explique le foudroiement de Sémélé par Zeus et toute une série de "cachettes » de Dionysos, dont nous avons montré ailleurs qu'elles pourraient bien être les étapes symboliques d'une immortalisation, entendons ici du recouvrement de son statut originel d'immortel ${ }^{64}$. C'est lors de cette quête que Dionysos impose à ceux qui ne reconnaissent pas sa divinité les cruelles pratiques du sparagmos et de l'ômophagie. Ce faisant, il rappelle son origine divine première, à savoir celui du Dionysos fils de Zeus et de Perséphone, démembré par les Titans, et, du même coup, il punit ceux qui ne reconnaissent en lui qu'une figure

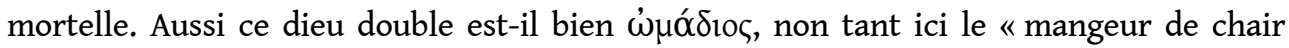
crue » que « celui qui fait manger la chair crue ».

En somme, pour en revenir au passage de Plutarque qui nous occupe, il y est question d'un même dieu, évoqué dans les différentes phases de sa geste, et, de la même manière, les initiés revivent son "drame», depuis son démembrement jusqu'à son «immortalisation» et la reconnaissance de sa place au sein des dieux. C'est au Dionysos reconnu dieu que sont adressées les cérémonies sur lesquelles Plutarque veut garder le silence, c'est au Dionysos en quête de cette "immortalité " que sont adressées les pratiques qu'il condamne, comme l'ômophagie. Ici, intervient la question de la distinction qu'établit Plutarque entre les $\delta \alpha i ́ \mu o v \varepsilon \varsigma$ qui se manifestent dans les mystères et les $\varphi \alpha \tilde{\lambda} \lambda$ or

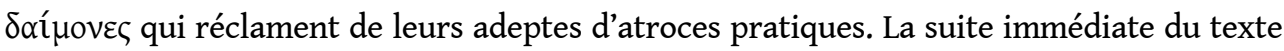
présente une distinction qui semble se superposer à celle-là : «Ce que l'on dit et que l'on chante dans les mythes et les hymnes, les rapts commis par les dieux, leurs errances, leurs cachettes, leurs exils, leurs servitudes, tout cela n'est pas le fait des dieux; ce sont des épreuves et des aventures arrivées aux démons $»^{65}$. On se trouve ici au cœur de la théorie démonologique grâce à laquelle Plutarque a rendu compte des cultes à mystères ${ }^{66}$. Ceux-ci font revivre le «drame » vécu par la divinité : il s'agissait dès lors d'expliquer comment un dieu peut être sujet aux passions sans perdre sa supériorité divine, à savoir son impassibilitée ${ }^{6}$. La réponse est la suivante : seuls les daimones sont passibles.

Les deux passages repris ci-dessus placent Dionysos dans une sorte de dynamique ascendante qui le mène du rang de $\varphi \alpha \tilde{\nu} \lambda$ os $\delta \alpha i ́ \mu \omega v$ à celui de $\delta \alpha i ́ \mu \omega v$ et enfin à celui de $\theta \varepsilon o ́ c$. Cette explication philosophique du culte s'appuie certainement sur une tradition mythique que ne pouvait méconnaître Plutarque: au terme d'une série d'épreuves relevant du parcours initiatique, Dionysos a bien sa place sur l'olympe, auprès des immortels ${ }^{68}$. Enfin reconnu dieu, il siège parmi les dieux de l'olympe, comme en témoigne notamment l'Hymne à Pan. Le poète y montre Hermès allant présenter son fils à l'assemblée des dieux : «Il s'assit auprès de Zeus et des autres immortels et leur présenta son enfant : tous les immortels se réjouirent en leur cœur, surtout Dionysos Baccheios " ${ }^{6}$. Cette épiclèse ( $\beta \alpha \kappa \chi \varepsilon \tilde{o}$ ou $\beta \alpha$ $\alpha \chi \circ \varsigma)$ n'est pas anodine. Si elle évoque sans doute 
immédiatement le dieu « qui agite et qui rend fou $»^{70}$, on constate qu'ici elle s'applique au dieu installé sur l'olympe : que peut-elle bien évoquer alors si ce n'est l'immortalité du dieu, comme, dans les Hymnes orphiques, elle est l'épiclèse que porte Dionysos quand, fils de Sémélé, il a recouvré son immortalité originelle, selon l'interprétation de J. Rudhardt ${ }^{71}$ ? La suite de notre réflexion pourrait bien la conforter.

En effet, ßó́kхoৎ est également le titre que s'attribue le chœur dans un fragment de la parodos des Crétois, tragédie perdue d'Euripide, conservé dans le De abstinentia de Porphyre ${ }^{72}$. Le philosophe, qui défend l'idée que la piété totale est de s'abstenir de manger toute chair animale ${ }^{73}$, invoque le témoignage d'Euripide à propos de prophètes crétois de Zeus observant cette abstinence. Le chœur s'adresse à Minos : «Je mène une vie pure depuis le jour où je devins un myste du Zeus de l'Ida, et que, ayant accompli les tonnerres de

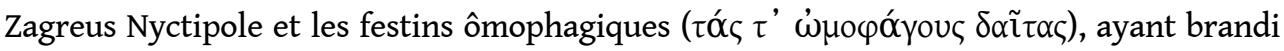
des flambeaux à la Mère des monts et ayant été purifié, appartenant aux Courètes ${ }^{74}$, je fus appelé bacchos. Revêtu d'habits blancs, je fuis la naissance des mortels, je ne m'approche pas des cercueils et je me garde de consommer des nourritures animées ${ }^{75}$.

Ce passage a suscité une abondante littérature tant sur les indices d'un éventuel contexte orphique évoquant Dionysos que sur le type de culte qui y est évoqué : Euripide évoque-til un seul rituel ou a-t-il réuni des éléments d'origine diverse? Le rituel dont il est question s'adresse-t-il à une ou plusieurs divinités ${ }^{76}$ ? La tentation fut grande de mettre ce fragment des Crétois en relation avec le récit évhémériste de Firmicus Maternus situant le démembrement de Dionysos en Crète ${ }^{77}$. A priori pourtant, un faisceau d'indices engage à privilégier l'hypothèse d'un culte à Zeus, plutôt qu'à Dionysos. D'abord, le chœur évoque explicitement des mystères en son honneur que l'on peut rapprocher des mystères auxquels aurait été initié Pythagore: "Arrivé en Crète, il se présenta aux mystes de Morgos, un des Dactyles de l'Ida, qui le purifièrent à l'aide de la pierre de foudre [...]. Descendu dans la grotte dite de l'Ida avec de la laine noire, il y passa les trois fois neuf jours rituels, sacrifia à Zeus, [...] » ${ }^{78}$. Ensuite, ni le nom de Zagreus ni l'épithète Nyctipole ne sont à l'usage exclusif de Dionysos. Outre que l'identification de Zagreus au Dionysos orphique est hasardeuse et tardive voire peu fondée ${ }^{79}$, Zagreus peut désigner d'autres divinités ${ }^{80}$ et rien ne permet d'écarter fermement l'hypothèse d'un Zeus-Zagreus ${ }^{81}$. Quant

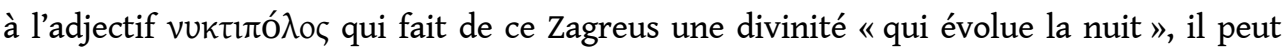
être appliqué à diverses divinités, souvent associées aux Enfers ${ }^{82}$, mais pas toujours ${ }^{83}$, et il évoque plus souvent l'ambiance nocturne dans laquelle se déroulait le rite ${ }^{84}$. La présence des Courètes est également liée à Zeus, et plus particulièrement au moment de sa naissance. Euripide est considéré comme la source la plus ancienne mentionnant les Courètes dans ce contexte ${ }^{85}$ et plus tard s'est forgé le thème d'une confrérie sacerdotale dont le culte bruyant couvrit les vagissements du petit Zeus caché par sa mère ${ }^{86}$. Cette dernière n'est d'ailleurs pas absente de ce texte: la "Mère des monts » évoque Cybèle, assimilée à Rhéa. C'est précisément dans les Bacchantes, que l'on trouve associés à deux reprises Cybèle, évoquée dans un cadre montagnard et assimilée à Rhéa la grande Mère, Zeus et les Courètes crétois ${ }^{87}$. Mais, comme ils avaient veillé sur Zeus enfant, les Courètes ont également protégé l'enfance de Dionysos, si l'on en croit Clément d'Alexandrie qui rapporte certainement une tradition plus ancienne ${ }^{88}$.

Jusqu'ici donc, aucun indice ne permet vraiment de trancher entre Zeus et Dionysos dans la question du dieu auquel sont destinées les pratiques mentionnées dans cet extrait. Reste le terme bacchos sur lequel il convient encore de revenir. 

dieu ßó́xxos s'applique également à l'initié qui, après avoir réactualisé le drame vécu par le dieu, est lui-même arrivé au terme de l'initiation ${ }^{89}$. Le fragment des Crétois, inséré par Porphyre dans un important développement sur la pureté, indique qu'à ce moment-là celui qui est bacchos est pur. Dans un autre contexte qui vient confirmer ce rapport à la pureté, un texte, certes tardif, mentionne une ancienne coutume athénienne qui consistait à expulser deux pharmakoi lors de la fête des Thargélies célébrée en l'honneur d'Apollon afin de purifier la cité : ces deux pharmakoi recevaient le nom de $\sigma 0 ́ \mu \beta \alpha \kappa \chi 0{ }^{90}$. Enfin, dans un usage métaphorique de la langue des mystères, Platon décrit l'arrivée chez Hadès de celui qui "purifié et initié, habitera avec les dieux », chose rare, ajoute-t-il, car "nombreux sont les porteurs de thyrse, rares sont les bacchants ${ }^{91}$ : purification, initiation, et enfin proximité avec les dieux, telles sont les étapes franchies par les bacchants pour atteindre une forme d'immortalisation, conférée par la compagnie des dieux.

Il faut également rappeler que le terme Bacchos ne renvoie pas systématiquement à la possession dionysiaque, même métaphoriquement ${ }^{92}$. Dionysos lui-même n'est pas désigné sous ce nom avant le $\mathrm{v}^{\mathrm{e}}$ siècle avant $\mathrm{J} . \mathrm{C}$., nom qui lui est donné à partir des manifestations que recouvre le verbe $\beta \alpha \kappa \chi \varepsilon \varepsilon_{\varepsilon i v}{ }^{93}$. Aussi d'autres dieux peuvent-ils recevoir cette épiclèse ${ }^{94}$. Est-ce le cas de Zeus? Une inscription mentionne en effet un

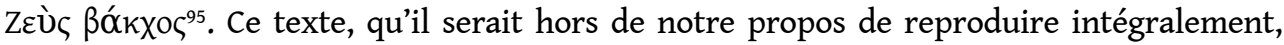
date du règne d'Antonin le Pieux et rapporte un oracle contenant des prescriptions rituelles destinées à éloigner une peste. Quatre divinités y sont clairement concernées. Elles sont évoquées à trois reprises : une première fois, sous les noms de Zeus, Athéna, Dionysos et Paeéôn ; une deuxième fois, sous les noms de Cronide, Eiraphiôtès (épiclèse de Dionysos), Tritogeneia (épiclèse d'Athéna) et d'Asclépios ; une troisième fois, sous les noms de Pallas, Zeus, Zeus Bacchos et de pais (épiclèse d'Asclépios). Le parallèle entre les trois séries de noms établit les équivalences suivantes : Zeus-Cronide-Zeus / Athéna-Tritogeneia -Pallas / Dionysos-Eiraphiôtès-Zeus Bacchos / Paeéôn-Asclépios-pais. Le doute n'est pas permis ici: Zeus Bacchos est bien un autre nom de Dionysos, soulignant peut-être sa proximité avec Zeus dont il se proclame le fils dès le premier vers des Bacchantes et avec qui il a en commun une enfance "cachée ", protégée par les Courètes crétois. Si cette inscription, éloignée d'Euripide dans le temps et dans l'espace, ne peut à elle seule ${ }^{96}$ confirmer à quel dieu, de Zeus ou de Dionysos, s'adressent les rites dont fait mention l'extrait des Crétois, l'hypothèse d'une atmosphère dionysiaque reste privilégiée.

Rien ne permet non plus d'affirmer que, dans une sorte de parcours initiatique, le dieu, qu'il s'agisse de Zeus ou de Dionysos, ait mangé de la chair crue, imposant ensuite semblable pratique à ses sectateurs. Aucune indication ne le prouve explicitement dans

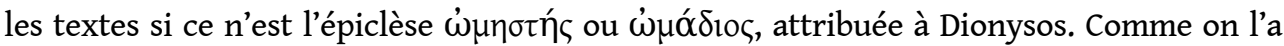
$\mathrm{vu}$, le rituel évoqué par Plutarque et par Porphyre suggère néanmoins clairement un rituel en deux temps correspondant au double statut du dieu : une première phase, dans laquelle est pratiquée l'ômophagie, serait destinée au dieu non encore reconnu dieu, suivie d'une seconde phase, d'où serait évacuée l'ômophagie, phase au terme de laquelle l'initié serait, comme le dieu, Bacchos. L'ômophagie pourrait donc bien être une pratique qui s'insère dans un culte destiné à un dieu "caché » et "immortalisé ». La piste nous semble intéressante en ceci que le changement de mode alimentaire est lié à un changement de statut, aussi bien pour l'homme que pour le dieu. On se souviendra que, dans l'Hymne qui lui est consacré, Hermès, lui aussi en quête d'une reconnaissance de son 
immortalité et avide de passer de l'ombre de l'antre maternel à la lumière de l'olympe, accomplit un acte qui est central dans sa geste $:$ il abat deux des vaches volées à Apollon. L'Hymne précise ensuite que, troublé par l'odeur des chairs rôties, il est pris par une envie de viande ${ }^{97}$. Envie qu'il réfrène car telle n'est pas la nourriture des dieux ${ }^{98}$. Mais c'est sans doute une autre histoire...

Nous devons encore déterminer si l'ômophagie consiste bien à dévorer les chairs crues d'animaux sauvagement déchiquetés.

\section{L'ômophagie : nourrir le dieu caché ?}

Dans le passage de Porphyre analysé ci-dessus, P. Bonnechere relève une contradiction puisque, s'abstenant de nourriture vivante, le myste assiste aux festins ômophagiques ${ }^{99}$. Ajoutons qu'il serait assez étonnant que Porphyre, qui invoque Euripide pour défendre le végétarisme, ait fait allusion à un extrait où il est question de manger de la viande crue. Nous avons proposé une première explication fondée sur l'idée d'un rituel en deux phases attribué à un dieu " caché » puis reconnu, et effectué par des initiés qui passeraient de la consommation à l'abstinence de chairs crues. Il en est une seconde, déjà avancée par d'autres et fondée notamment sur le dossier épigraphique repris ci-dessous, à savoir qu'on pourrait rattacher l'ômophagie à une réalité moins cruelle que celle du manger cru et considérer cette dernière comme mythique ${ }^{100}$. Comme on le verra, loin d'être incompatible avec la première explication, elle vient au contraire la renforcer.

On trouve le terme $\omega$ Hopó $\gamma ı v$ dans une inscription appartenant à un type de document bien connu dans l'Asie Mineure et les îles de la mer Égée à l'époque hellénistique puisqu'il s'agit d'un contrat de vente de sacerdoce, pratique répandue dans ces régions depuis le IV e siècle. En l'occurrence, il s'agit d'une inscription datant des années 276/5 et concernant la vente de la prêtrise de Dionysos à Milet. Les quatre premières lignes stipulent ceci : «lorsque la prêtresse accomplit les hiera au nom de toute la cité, qu'il ne soit permis à personne de lancer l'ômophagion avant que la prêtresse ne l'ait lancé au nom de la cité, qu'il ne soit permis à personne non plus de rassembler le thiase avant le thiase public $»^{101}$. Suivent d'autres indications sur l'organisation du culte dionysiaque milésien, qui n'intéressent pas directement notre propos, mais qui soulignent le caractère officiel du culte, tout en suggérant que des thiases privés pouvaient être rassemblés à condition que le thiase officiel soit rassemblé en premier ${ }^{102}$ : sont évoqués les actes sacrificiels en l'honneur d'un Dionysos qui n'est pas autrement qualifié dans l'inscription, l'initiation à Dionysos qualifié de Bacchios, et, enfin, la fête des Katagôgia, escorte de Dionysos conduite par la prêtresse, et, comme on l'apprend là, le prêtre, enfin le retour (« descente ») à la cité $^{103}$. Afin de tenter d'identifier l'acte posé par la prêtresse, lisons une autre inscription de Milet, épitaphe d'une prêtresse de Dionysos, datée des $\mathrm{III}^{\mathrm{e}}-\mathrm{II}^{\mathrm{e}}$ siècles avant notre ère, et souvent mise en relation avec l'inscription précédente : « Vous avez dit adieu à la sainte prêtresse, Bacchantes citoyennes : juste hommage rendu à une noble femme. C'est elle qui vous menait à la montagne et qui, marchant à la tête de toute la cité, transportait tous les orgia et les hiera. Si un étranger demande son nom, c'est Alkméonis, fille de Rhodios, qui connaît la destinée des bons $»^{104}$.

Les deux inscriptions peuvent être comprises comme suit. La seconde, contemporaine ou

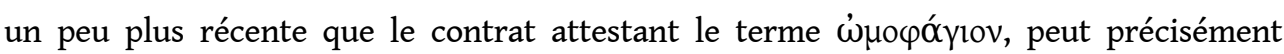
apporter un éclairage aux premières lignes de celui-ci. Il s'agit manifestement de

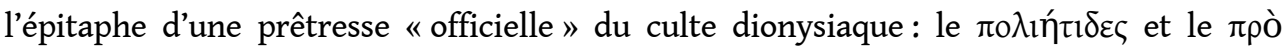


$\pi 0 ́ \lambda \varepsilon \omega \varsigma$ ne permettent guère d'en douter. Elle était donc la prêtresse chargée de lancer l' ômophagion en priorité et de rassembler le thiase. Cette épitaphe place ces gestes rituels dans la pratique bien attestée du ménadisme : la prêtresse emmenait les bacchantes vers la montagne. Il y est stipulé qu'elle transportait les orgia et les hiera. Soit elle le fait lors de cette course vers la montagne, soit dans une autre séquence d'actions. Quoi qu'il en soit, le verbe $\varphi \varepsilon ́ \rho \omega$ implique que ces hiera (terme générique) et ces orgia (terme à la connotation mystérique évidente) désignent des objets liés à la pratique du culte dionysiaque, et non des cérémonies ou des rites ${ }^{105}$.

Les deux inscriptions attestent bien les activités essentielles de la prêtresse de Dionysos à Milet et, se complétant mutuellement, permettent de reconstituer les étapes du rituel comme suit : réunion d'un thiase, oribasie féminine, procession civique, avec le transport en grande pompe des insignes du culte du dieu, prêtresse en tête, et retour à la cité, le

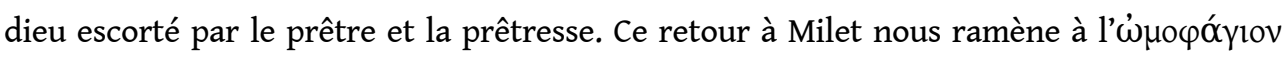
$\dot{\varepsilon} \mu \beta \alpha \lambda \varepsilon \tilde{v} v$ de la première inscription. De quel acte rituel s'agit-il ? Et où se place-t-il dans la séquence reconstituée ci-dessus? Le verbe exprime très concrètement l'action de déposer, de jeter quelque chose dans autre chose. Il est abondamment attesté dans les inscriptions quand il s'agit de verser une somme d'argent dans le thesauros d'un sanctuaire, ou dans le cas d'offrandes jetées dans un trou, dans un fleuve ou dans la mer. Cette action est évoquée, précisément dans un contexte dionysiaque, dans le Papyrus de Gourob, daté du $\mathrm{III}^{\mathrm{e}} \mathrm{s}$. avant J.-C ${ }^{106}$. Ce document, mutilé, conserve peut-être des i $\varepsilon \rho o \grave{~}$ 入óyol rédigés à la demande de Ptolémée Philopatôr. Y sont invoquées différentes divinités, Brimô, Déméter Rhéa, les Courètes. Il y est fait mention du sacrifice d'un bélier et d'un bouc, avec consommation sur place de la viande. Suivent encore une invocation à Eubouleus et Pallas, l'énumération des symbola de Dionysos, la mention du dieu dia kolpos (Sabazios ?), d'un boukolos, d'une formule rituelle (synthèma), et de ce qui a été « donné "

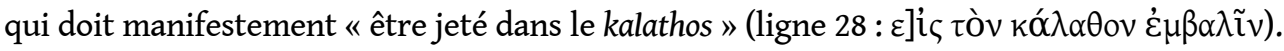
Enfin sont mentionnés des objets, cône, toupie, osselets, miroir, rappelant les jouets utilisés par les Titans pour attirer le petit Dionysos dans un piège. Certes le papyrus ne précise pas l'objet du lancer, mais il y est clairement question de jeter dans une corbeille.

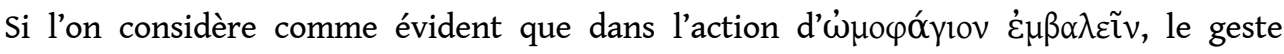
évoqué par le verbe $\dot{\varepsilon} \mu \beta \alpha \lambda \varepsilon \tilde{v} v$ neutralise la notion de manducation présente dans le terme

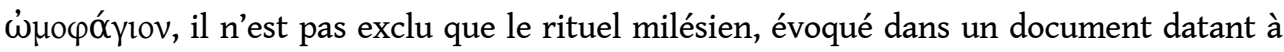
peu près de la même époque, fasse allusion à une « précipitation dans une corbeille » d'un morceau de viande crue, comme l'avait déjà suggéré Albert Henrichs ${ }^{107}$. Par conséquent, le destinataire ne peut être une ménade en transe, mais bien le dieu lui-même. C'est alors

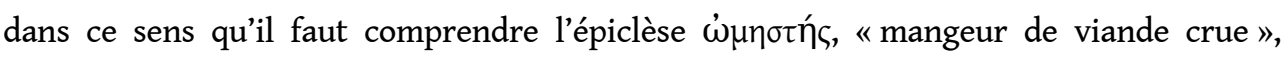
attribuée à Dionysos.

Enfin, même si, méthodologiquement, il est difficile de considérer que ce qui se passait à Milet vaut pour tous les lieux et tous les temps, une mise en perspective de ces sources épigraphiques et papyrologiques avec les textes de Plutarque et de Porphyre, et plus particulièrement avec le passage des Crétois cité par ce dernier, permettrait d'entrevoir à nouveau un rituel en deux temps et deux lieux. L'état lacunaire de l'inscription, dont on ne sait si elle atteste les différentes séquences d'un même rituel ou des actes indépendants les uns des autres, ne peut malheureusement ni confirmer ni infirmer notre hypothèse. Il n'en reste pas moins qu'elle suggère un passage de la nuit au jour (l. $23 \pi \rho$

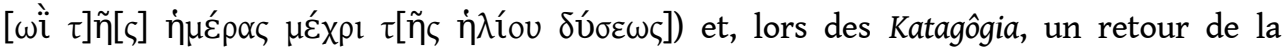
montagne (lieu sauvage) à la cité (lieu civilisé). Ce rituel s'accorderait ainsi aux données 
du mythe. En effet, comme nous l'avons montré dans notre recherche sur les pratiques immortalisantes, ce passage de l'ombre à la lumière va de pair avec un changement de statut et le cas de Dionysos est, à cet égard, tout à fait représentatif. Dieu caché avant d'être reconnu dieu, Dionysos l'est notamment dans un coffre ( $\left.\lambda \alpha \alpha^{\prime} \rho{ }^{\alpha} \xi^{108}\right)$. C'est là un des multiples contenants associés au dieu aux côtés de la ciste et du kalathos, sans pour autant que ces objets lui soient spécifiques. La ciste est une corbeille fermée ${ }^{109}$ destinée à contenir des vivres dans un contexte profane ${ }^{110}$ ou religieux ${ }^{111}$. Elle est évoquée avec le

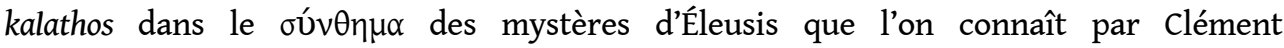

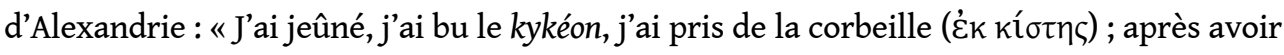

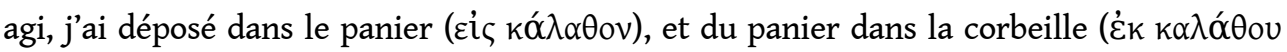

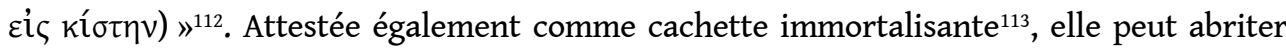
un serpent ${ }^{114}$ et semble avoir été liée à cet animal au point que le scholiaste d'Aristophane

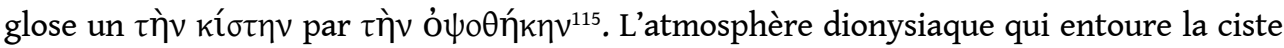
de Dicéopolis, le héros des Acharniens, est explicite. Il s'agit de la ciste "mystique" dont Nonnos, bien plus tard, reconstituera l'aition, attribuant son inventionà Mystis, servante d'Ino à qui fut confié l'enfant Dionysos ${ }^{116}$. Or, Dionysos est associé à différents animaux dont il peut parfois revêtir la forme : taureau et serpent sont les plus récurrents ${ }^{117}$. Le chœur des Bacchantes rappelle que c'est un dieu aux cornes de taureau que Zeus engendra et qu'il le couronna de serpents ${ }^{118}$. Plus loin, il l'invite à paraître à ses yeux sous la forme d'un taureau, d'un serpent ou d'un lion ${ }^{119}$.

Dans un contexte dionysiaque, ciste et serpent ne semblent avoir été rapprochés que tardivement, aux époques hellénistique et romaine, notamment sur des monuments figurés ${ }^{120}$, et dans un passage de Clément d'Alexandrie d'un grand intérêt pour notre

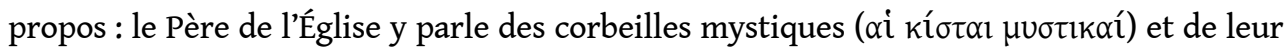
contenu, gâteaux, grains de sel et serpent, ópyıv de Dionysos Bassaros ${ }^{121}$. Le rapprochement avec l'inscription de Milet est suggéré par ceci: Dionysos Bassaros, épiclèse qui lui vient de la $\beta \alpha \sigma \sigma \alpha ́ \alpha \alpha$, tunique des Bacchantes lydiennes ${ }^{122}$, est caché symboliquement dans une ciste, sous la forme d'un serpent, désigné par le terme ópyıv du dieu, ce qui nous renvoie aux orgia transportés par la prêtresse de Dionysos de l'épitaphe ci-dessus. On pourrait ainsi voir dans ces témoignages tardifs l'aboutissement d'une fusion d'éléments dionysiaques d'origine et de tradition diverses. Il n'en reste pas moins que nous rencontrons, dès l'époque classique, quatre données qui peuvent être rapportées à Dionysos et qu'il est intéressant de confronter: la ciste, le serpent, une formule mystérique (rapportée par clément mais rendant certainement compte d'une pratique plus ancienne) faisant allusion à la ciste et au kalathos, et, sinon le geste de l' ômophagion emballein, en tout cas l'allusion à l'ômophagie. Les rapprochements avérés entre mystères éleusiniens etbachiques ${ }^{123}$ autorisent à repartir des gestes que suppose la formule éleusinienne : il y est question d'une sortie de la ciste, d'un dépôt dans le kalathos, puis d'un retour du kalathos dans la ciste. Aussi suggérons-nous l'hypothèse suivante. Le dieu est caché dans la ciste sous une forme animale (réelle ou métaphorique), tandis que le kalathos abrite un morceau de viande crue qu'on y aurait lancé, ciste au serpent et kalathos appartenant aux objets transportés par la prêtresse. Il s'agirait alors de sortir l'animal divin de la ciste, de le nourrir dans le kalathos avant de lui faire réintégrer la ciste. Ce geste concilie ainsi la pratique rituelle avec le régime des Grecs évoqué ci-dessus, révélant du même coup toute l'ambiguïté du dieu: animal quand il reçoit l'offrande de

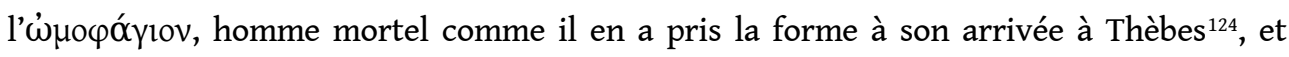
enfin dieu siégeant dans la lumière de l'olympe. 


\section{Conclusion}

L'analyse des sources littéraires et épigraphiques a montré combien il est difficile de trouver trace d'une pratique ômophagique au sens propre du terme. Rien ne permet d'affirmer non plus que nous avons affaire à un adoucissement d'une pratique ancienne, violente et sauvage. Au terme de cette enquête, il ne s'agit certes pas de dédouaner les Grecs d'un rituel qui pourrait nous paraître choquant, mais bien de le situer au plan des représentations. La présence de cette pratique dans les textes mythiques et le sens qu'on peut lui donner dans la pratique rituelle par la confrontation des sources littéraires et épigraphiques ont quelque chose à dire des représentations que les Grecs se faisaient de ce dieu. Il est possible que certains dieux aient reçu de la viande crue en certaines circonstances. Dans le cas de Dionysos, cette offrande devait surtout rappeler son image de dieu venu d'ailleurs, dieu marginal, à la limite de la bestialité, de l'humanité et du divin, et par là, toujours potentiellement dangereux, mais nécessaire pour l'équilibre des cités.

\section{NOTES}

1. M. HALM-TISSERANT, « Le sparagmos, un rite de magie fécondante », Kernos 17 (2004), p. 119-142. Ce laconisme avait déjà été relevé par Alb. Henrichs («Greek Maenadism from Olympias to Messalina ", HSCPh 82 [1978], p. 151).

2. HALM-TISSERANT, l.c. (n. 1), p. 134.

3. Dans une perspective structuraliste, l'ouvrage de Claude Lévi-Strauss (Mythologiques. Le cru et le cuit, Paris, Plon, 1964) a ouvert la voie aux études menées plus précisément dans le monde grec sur un sujet qui s'inscrit dans la recherche plus large des critères visant à cerner le degré de civilisation ou d'humanité d'un homme ou d'une société. Voir notamment Ch. SEGAL, "The Raw and the Cooked in Greek Literature : Structure, Values, Metaphor », CJ 69, 4 (1974), p. 289-308, ainsi que les contributions de J.-P. Vernant et M. Detienne auxquelles nous nous référons plus loin dans cet article. Ces études superposent au couple antinomique cru/cuit celui de sauvagerie/ civilisation ou encore de bestialité/humanité. Le dossier qui suit va dans ce sens mais n'implique pas que la pratique dionysiaque consiste en un retour périodique de la civilisation à une cruauté primitive, comme certains ont pu l'affirmer (voir HENRICHS, l.c. [n. 1], p. 123, qui a souligné cette dérive de l'interprétation structuraliste).

4. Aristophane, Cavaliers, 260.

5. Hippocrate, Du régime, texte établi et traduit par R. Joly (CUF), notice p. XVII et Xx.

6. Hippocrate, Du régime LVI, 3.

7. Ibid. LV, 5.

8. Plutarque, Du contrôle de la colère, 13 (Moralia, 461b).

9. Si le sacerdoce du flamine de Jupiter, qui a suscité chez l'auteur grec huit de ses Questions romaines, devait paraître original à ses yeux, c'est bien la tradition philosophique grecque et les usages de la pratique sacrificielle grecque qui sous-tendent sa réflexion (voir Plutarque, Grecs et 
Romains en parallèle, introduction, traduction et commentaires des Questions romaines et des Questions grecques par M. NOUILHAN, J.-M. PAILlER et P. PAYEN, Paris, 1999, p. 229-231).

10. Plutarque, Étiologies romaines, 109 (Moralia, 289e-f).

11. Ibid., 110 (Moralia, 289f-290a).

12. J.-P. VERNANT, «À la table des hommes. Mythe de fondation du sacrifice chez Hésiode », in M. DETIENNE et J.-P. VERNANT (éd.), La cuisine du sacrifice en pays grec, Paris, 1979, p. 70, n. 3.

13. Pour l'ensemble du passage, voir Julien, Contre les cyniques ignorants, 12 (191c-193c).

14. Eschyle, Suppliantes, 187.

15. Iliade IV, 35.

16. Iliade XXII, 347.

17. Iliade XXIV, 207.

18. Cet adjectif - ainsi que $\omega \mu$ ó́ $\delta ı \iota_{-}$- est également une épiclèse de Dionysos. Nous y reviendrons plus loin.

19. Xénophon, Anabase IV, 8, 14.

20. Voir Aristote, Histoire des animaux, 609b1, b4, b13, 610a 14, 615a4, 693a13, etc., ou encore, dans des images ou des comparaisons, Iliade V, 783 ; VII, 256 ; XI, 479 ; XV, 592 ; XVI, 157, etc.

21. Iliade XI, 454 ; XXII, 67.

22. Thucydide, III, $94,5$.

23. Lucien, Histoire vraie I, 35.

24. Hérodote, IV, 106.

25. Eschyle, Sept contre Thèbes, 541.

26. Euripide, Phéniciennes, 1025.

27. Hésiode, Théogonie, 311.

28. Théognis, Poèmes élégiaques I, 542.

29. Lucien, Dialogues marins I, 5.

30. Lucien, Histoire vraie I, 3.

31. Porphyre, De abstinentia I, 13, 4 .

32. M. DETIENNE, Dionysos mis à mort, Paris, Gallimard, $1998^{2}$ [1977], p. 141.

33. À ce sujet, voir M. DETIENNE, Les jardins d'Adonis. La mythologie des parfums et des aromates en Grèce, nouvelle édition augmentée d'un après-propos de l'auteur, Paris, Gallimard, 1972, p. 29-34. 34. Voir l'interprétation de J.-P. Vernant qui fait suite à l'ouvrage de M. Detienne cité à la note précédente, p. 242-243. Sur cette tension entre le cru et le cuit, c'est-à-dire entre le sauvage et le civilisé, voir encore récemment M. HERRERO DE JAUREGUI, «Dionysos mi-cuit: l'étymologie de Mésatis et le festin inachevé des Titans », RHR 223, 4 (2006), p. 395-401.

35. Voir notamment HENRICHS, l.c. (n. 1), p. 150-151 ; Dirk OBBINK, « Dionysus Poured Out : Ancient and Modern Theories of Sacrifice and Cultural Formation », in Th.H. CARPENTER, Chr. A. FARAONE (éds.), Masks of Dionysus, Ithaca / London, Cornell University Press, 1993, p. 69-75 ; M. DILLoN, Girls and Women in Classical Greek Religion, London / New York, Routledge, 2003, p. 142-143 ; HALMTISSERANT, l.c. (n. 1), p. 128 ; B. GOFF, Citizen Bacchae. Women's Ritual Practice in Ancient Greece, Berkeley / Los Angeles / London, University of California Press, 2004, p. 272.

36. Euripide, Bacchantes, 1242.

37. Ibid., 135-138.

38. Ibid., 735-747.

39. Pseudo-Apollodore, Bibliothèque III, 37.

40. Voir à ce sujet I. TASSIGNON, «Vingt mille lieux sous les mers avec Dionysos et Télibinu », in Éd. DELRUELLE et V. PIRENNE-DELFORGE (éd.), Kĩnol. De la religion à la philosophie. Mélanges offerts à André

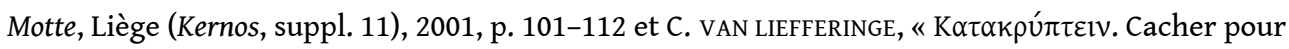
immortaliser », LEC76 (2008), p. 107-125.

41. Euripide, Bacchantes, 254, 272, etc. 
42. Ibid., 482.

43. Ibid., 242-245.

44. H. Jeanmaire, Dionysos. Histoire du culte de Bacchus: l'orgiasme dans l'Antiquité et les temps modernes, origine du théâtre en Grèce, orphisme et mystère dionysiaque, évolution du dionysisme après Alexandre, Paris, $1970^{2}$ [1951], p. 153-155.

45. Lucien, Bacchos, 2, 21.

46. Voir ci-dessus.

47. Voir la banque de données électronique des épiclèses grecques du CRESCAM qui signale pour

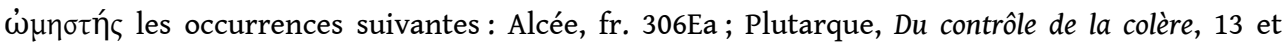
Antoine, 24, 2; Phainias apud Plutarque, Thémistocle, 13 Pélopidas, 21, et Aristide, 9; et pour $\omega\lrcorner \mu \alpha ́ \delta 10 \varsigma$ les occurrences suivantes: Hymnes orphiques,30, 5 et 52, 7 ; Euelpis de Carytos, FHG IV, $408=$ Porphyre, De abst. II, 55, 3 = Eusèbe, Prép. Év. IV, 16, 5.

48. Hymnes orphiques, 30, 5 (A.-Fr. MORAND, Études sur les Hymnes orphiques, Leiden, Brill, 2001, p. 13).

49. MORAND, o.c. (n. 48), p. 150-151.

50. P. BONNECHERE, Le sacrifice humain en Grèce ancienne, Liège (Kernos, suppl. 3), 1994, p. 288-291. À ce sujet, voir également S. LEBRETON, "Dionysos Ômèstès (Plutarque, Thémistocle, 13; Antoine, 24)", in L. Bodiou, V. MEHL, J. OUlHen, Fr. PRost, J. Wilgaux (éd.), Chemin faisant. Mythes, cultes et société en Grèce ancienne. Mélanges en l'honneur de Pierre Brulé, Rennes, Presses Universitaires, 2009, p. $195-197$.

51. Eusèbe de Césarée, Prép. év. IV, 16, 5.

52. Voir la note 4 de la page 118 de J. Bouffartigue et M. Patillon (CUF).

53. Porphyre, De abstinentia II, 55, 3.

54. BONNECHERE, o.c. (n. 50), p. 225.

55. S. Georgoudi (« Sacrificing to Dionysos : Regular and Particular Rituals », in R. SCHLESIER (éd.), A Different God? Dionysos and Ancient Polytheism, Berlin-Boston, De Gruyter, 2011, p. 50) a relevé la confusion qui a parfois été faite entre Dionysos Omadios ou Omèstès d'une part, et Dionysos Anthroporrhaistès de l'autre, et qui a amené à parler de cannibalisme. Invitant à la prudence dans l'interprétation de ces rituels, elle rappelle encore qu'on a parfois considéré que manger la chair crue d'un animal revenait pour le fidèle à manger la chair et le sang du dieu afin d'en incorporer les vertus (p. 51)

56. HENRICHS, l.c. (n. 1), p. 150-151.

57. É. COCHE DE LA FERTÉ, «Penthée et Dionysos. Nouvel essai d'interprétation des Bacchantes d'Euripide », Recherches sur les religions de l'Antiquité Classique, Paris, 1980, p. 135-137.

58. BONNECHERE, o.c. (n. 50), p. 211.

59. Plutarque, De defectu oraculorum, 14 (Moralia, 417c).

60. Pindare fr. $70 b$ (dithyr. 2), 13-14 Snell.

61. Plutarque, Consolation à sa femme, 611d et la note 2 de la page 197 (p. 256) de J. Hani (CUF).

62. Voir le texte cité ci-dessous à la note 65 , dans lequel Porphyre fait explicitement allusion aux « errances » et aux « cachettes » du dieu.

63. J. RUDHARDT, « Les deux mères de Dionysos, Perséphone et Sémélé, dans les Hymnes orphiques ", RHR 219, 4 (2002), p. 483-501, citation de la page 493. Voir également J. RUDHARDT, Opera inedita. Essai sur la religion grecque et Recherches sur les Hymnes orphiques, édités par Ph. BORGEAUD et V. PIRENNE-DELFORGE, Liège (Kernos, suppl. 19), 2008, p. 268-272.

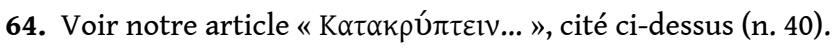

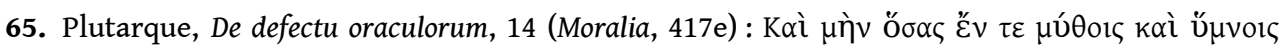

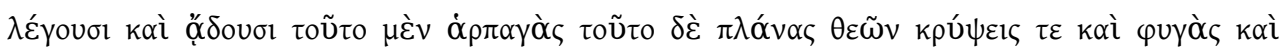

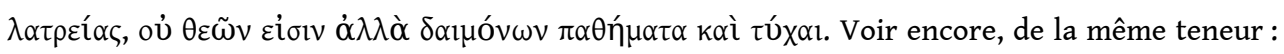
Plutarque, Isis et Osiris, 25 (Moralia, 360e-f). 
66. À ce sujet, voir Andrei timotin, La démonologie platonicienne. Histoire de la notion de daimōn de Platon aux derniers néoplatoniciens, Leiden, Brill, 2012, p. 164-204.

67. La question de l'impassibilité des dieux et des êtres supérieurs, soulevée, comme on le voit ici, par les philosophes du moyen-platonisme, sera au cœur des débats qui animeront l'école néoplatonicienne : voir notamment Jamblique, Réponse à la Lettre de Porphyre à Anébon, 34 à 45, où le philosophe répond à cette question par le statut de la théurgie.

68. Si Dionysos devait se faire reconnaître comme dieu par les hommes, il devait également se faire une place auprès des Olympiens : c'est ce que montre l'Hymne 1 à Dionysos dans sa version complète et récente (M.L. WeST, The Homeric Hymns, Homeric Apocrypha, Lives of Homer, Cambridge, Massachusetts (Loeb Classical Library), 2003) : voir M. WEST, « The first Homeric Hymn to Dionysos », in A. FAULKNER (éd.), The Homeric Hymns. Interpretative Essays, Oxford, University Press, 2011, p. 3031.

69. Hymne à Pan, 44-46.

70. Hérodote, IV, 79.

71. Voir l'étude de J. Rudhardt citée ci-dessus.

72. Porphyre, De l'abstinence, tome III, livre IV, texte établi, traduit et annoté par M. PATILLoN et A. Ph. SEGONDS, avec le concours de L. BRISSON, Paris, Les Belles Lettres (CUF), 1995, notice p. XLIIIXLVI ; Euripide, tome VIII, $2^{\mathrm{e}}$ partie, Fragments. Bellérophon - Protésilas, texte établi et traduit par F. JOUAN et H. VAN LOOY, Paris, Les Belles Lettres (CUF), 2000.

73. On ne s'étonnera pas de trouver une même veine chez Plutarque, adversaire de l'alimentation carnée, et chez Porphyre, défenseur du végétarisme. À ce sujet, voir F. JOURDAN, " Porphyre, lecteur et citateur du traité de Plutarque Manger de la viande », REG 118 (2005), p. 426435.

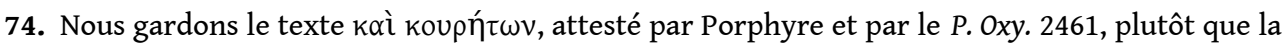

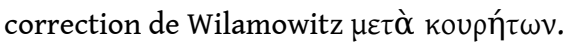

75. Porphyre, De abstinentia IV, 19.

76. F. Jouan et H. Van Looy (voir ci-dessus, n. 72) font le point sur ces questions (p. 310 et p. 324, n. 44).

77. Firmicus Maternus, Sur l'erreur des religions paiennes VI, 1-5. C'est le lien qu'établit H. Jeanmaire (o.c. [n. 44], p. 257). Sur la présence du culte de Dionysos en Crète, voir H. VERBRUGGEN, Le Zeus crétois, Paris, Les Belles Lettres, 1981, p. 119-121.

78. Porphyre, Vie de Pythagore, 17.

79. Voir à ce sujet JEANMAIRE, o.c. (n. 44), p. 408.

80. Ainsi, dans un fragment du Sisyphe d'Eschyle, il désigne le fils d'Hadès (fr. 228 Sommerstein).

81. Si H. Verbruggen (o.c. [n. 77], p. 122) souligne l'incertitude de l'identification de Zagreus et remet en question le sens de "Grand Chasseur » souvent donné au mot, il n'évacue pas de cet extrait le dionysisme dans lequel, selon lui, Euripide situe ici Zagreus (p. 124-125) reprenant, au sujet de ce passage, la conclusion de H. Jeanmaire (o.c. [n. 44], p. 272).

82. Il peut s'agir d'Hécate (Euripide, Ion, 1049 ; Apollonios de Rhodes, Argonautiques III, 862 ; IV, 148 etc.).

83. C'est le cas de Thémis dans les Hymnes orphiques $(79,7)$.

84. Qu'il s'agisse des courses des Bacchantes sur la montagne (Euripide, Ion, 717) ou, plus tardivement et sur un mode satirique, des mystères nocturnes institués pour le devin Protée (Lucien, De morte Peregrini, 29).

85. Euripide, Bacchantes, 120. Voir VERBRUGGEN, o.c. (n. 77), p. 46.

86. Tradition présente chez Callimaque, Hymne à Zeus, 52-54 et dont Virgile est le relai le plus célèbre dans la tradition latine (Géorgiques IV, 150-152).

87. Euripide, Bacchantes, 81-82 et 121.

88. Clément d'Alexandrie, Protreptique II, 17, 2. 
89. Relevé également par S.G. ColE, "Finding Dionysos", in D. OGDEN, A Companion to Greek Religion, Oxford, Blackwell, 2007, p. 327.

90. Helladios apud Photium, Bibliothèque, 279, p. 182, 6-7 (CUF). Voir BONNECHERE, o.c. (n. 50), p. 297.

91. Platon, Phédon, 69c. La phrase de Platon renvoie à Musée et Eumolpos, donc aux Eumolpides d'Éleusis. Voir G. PUGLIESE-CARRATELli, « Ancora sulla lamina orfica di Hipponion », PP 31 (1976), p. 463.

92. C'est l'idée que défend G. Pugliese-Carratelli qui, pour la question qui nous occupe ici, avance que rien ne permet d'affirmer le caractère dionysiaque du terme ßókxoৎ qui désigne dans ce fragment les mystes de Zagreus, pas même le parallèle pourtant étroit entre la triade des dieux

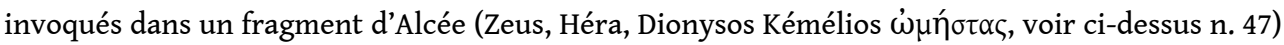
et les dieux du fragment des Crétois (Zeus, Mère des Monts, Zagreus) (l.c. [n. 91], p. 465-466).

93. JEANMAIRE, o.c. (n. 44), p. 58-59.

94. Voir G. PUGLIESE-CARRATELli, l.c. (n. 91), p. 463.

95. I. Pergamon, 324 = G. KAIBEL, Epigrammata Graeca exlapidibus conlecta, Berlin, 1878, p. 450-451.

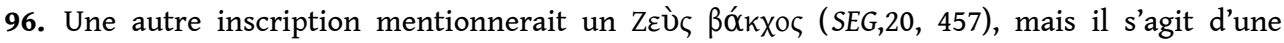
inscription mutilée mentionnant un $\Delta \grave{\imath} \beta \alpha \kappa[$ restitué en $\Delta$ ì $\beta \alpha ́ k \chi \omega l$, restitution contestée par J. et L. Robert (Bulletin épigraphique 1964-1967, p. 234, no 516).

97. Hymne à Hermès, 112-141.

98. Au sujet de la séparation que peut instituer la nourriture entre mortels et immortels, G. Ekroth invite cependant à nuancer, rappelant que dans certains récits mythiques ou certains rituels les dieux ne se repaissent pas que de la knisè. Elle mentionne notamment le mythe de Tantale dans lequel Déméter dévore l'omoplate de Pélops et, dans la pratique cultuelle, le rite des trapezomata, offrandes de viande crue placées sur une table ou l'autel du sacrifice, et destinées ensuite aux prêtres et aux prêtresses. Elle reconnaît toutefois que cette dernière pratique, peu documentée pour la période archaïque, a été suggérée et développée à partir de la coutume qui consistait précisément à réserver des parts au personnel du culte (G. ЕКROTH, «Burnt, cooked or raw? Divine and human culinary desires at Greek animal sacrifice », in E. STAVRIANOPOUlou, A. michaels, Cl. ambos (éd.), Transformations in Sacrificial Practices. From Antiquity to Modern Times, Berlin, 2008, p. 87-111, et particulièrement p. 97-98 et 104).

99. BONNECHERE, o.c. (n. 50), p. 146, n. 626.

100. A.J. FESTUGière, "Omophagion emballein », Études de religion grecque et hellénistique, Paris, Vrin, 1972, p. 110-113. Pour d'autres références, voir ci-dessus, la n. 35.

101. LSAM 48, 1-4 (reproduite par A.-Fr. JACCOTTET, Choisir Dionysos. Les associations dionysiaques ou

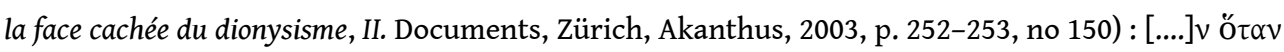

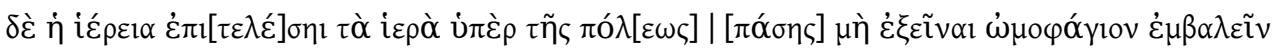

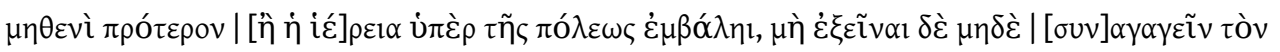

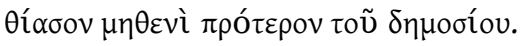

102. Comme le souligne S.G. Cole en se fondant précisément sur ces inscriptions, la frontière entre culte privé et culte public est difficile à situer (l.c. [n. 89], p. 337). À ce sujet, voir JACCOTTET, o.c. (n. 101), I. Texte, p. 75 et « Du thiase aux mystères. Dionysos entre le « privé » et « l'officiel »,

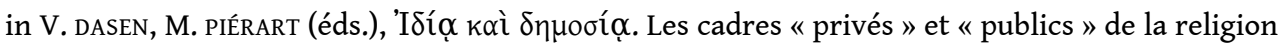
grecque antique, Liège, 2005 (Kernos, suppl. 15), p. 193-195. Dans cette dernière contribution, l'auteur avance l'hypothèse d'une création a posteriori du thiase « officiel », tout en soulignant à juste titre que la démarcation entre privé et public n'est pas stricte du point de vue de l'acte rituel proprement dit. A.-Fr. Jaccottet avait déjà mis en lumière le double visage de Dionysos, « dieu a-politique qui a sa place dans la cité auprès des individus » («Le lierre de la liberté », ZPE 80 [1990], p. 154-156). Sur cette question, traitée par le biais de l'iconographie, voir Cl. BÉRARD et Chr. BRON, «Bacchos au cœur de la cité. Le thiase dionysiaque dans l'espace politique », in 
L'association dionysiaque dans les sociétés anciennes, Actes de la table ronde organisée par l'École française de Rome (Rome 24-25 mai 1984), Rome, 1986, p. 13-30.

103. Ce rituel, attesté par l'iconographie dès le $\mathrm{vI}^{\mathrm{e}}$ siècle, est considéré comme une invitation à faire revenir une divinité rebelle, voire hostile, auprès des hommes, retour se faisant par voie maritime (voir I. TASSIGNON, «Dionysos et les Katagôgies d'Asie Mineure », in A. MOTTE, Ch.M. TERNES (éd.), Dieux, fêtes, sacré dans la Grèce et la Rome antiques, Actes du Colloque tenu à Luxembourg du 24 au 26 octobre 1999, Turnhout, Brepols, 2003 [Homo Religiosus II, 2], p. 81-99). Si notre inscription ne suggère pas un retour par voie d'eau mais une descente vers la cité, en revanche elle conforte l'idée d'une "réintégration progressive d'une divinité exclue du monde des hommes » (TASSIGNON, l.c., p. 97), c'est-à-dire, de notre point vue, d'une divinité cachée.

104. Inschriften von Milet II, 733 (reproduite par JAccotTET, o.c. [n. 101], p. 250-251, no 149) : Tìv

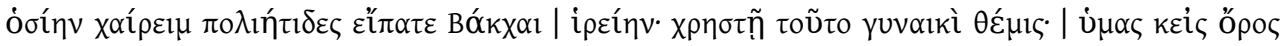

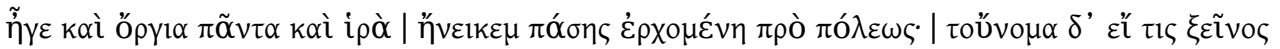

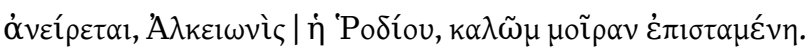

105. Voir A. MOTTE et V. PIRENNE-DELFORGE, « Le mot et les rites. Aperçu des significations de ópүla et de quelques dérivés », Kernos 5 (1992), p. 128-130.

106. P. Gurôb $1=$ Pack $^{2}$ 2464. Réédité et commenté par J.H. HORDERN, « Notes on the Orphic Papyrus from Gurôb (P. Gurôb 1 ; Pack 2464) », ZPE 129 (2000), p. 131-140.

107. HENRICHS, l.c. (n. 1), p. 150-151.

108. Pausanias, III, $24,3$.

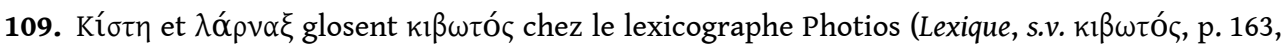
15 Porson).

110. Odyssée VI, 76.

111. Il peut s'agir de l'offrande d'un gâteau à Déméter (Aristophane, Thesmophories, 284-285) ou d'un repas dans le contexte dionysiaque des Anthestéries (Aristophane, Acharniens, 1086 et 1098).

112. Clément d'Alexandrie, Protreptique II, 21, 2.

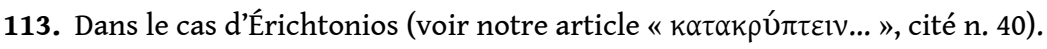

114. Voir encore le cas d'Érichtonios. Cette présence du serpent dans une ciste se rencontre dans des contextes autres que dionysiaques qui révèlent néanmoins la récurrence de cette association (voir notamment Aristophane, fr. 28 Henderson; Plutarque, Alexandre, 85, 2).

115. Scholia in Acharnenses, 1086.

116. Nonnos, Dionysiaques IX, 127.

117. Sur ce côté animal du dieu et ses implications rituelles, voir E. ASTON, Mixanthrôpoi. Animalhuman hybrid deities in Greek religion, Liège, 2011 (Kernos, suppl. 25), p. 127-130.

118. Euripide, Bacchantes, 99-101.

119. Ibid., 1018-1019. Cette présence récurrente du serpent dans les rites et les mythes dionysiaques a été retenue par Clément d'Alexandrie qui, dans une vision chrétienne, voit dans l'animal l'incarnation du mal et de la luxure (à ce sujet, voir F. JOURDAN, «Dionysos dans le Protreptique de Clément d'Alexandrie. Initiations dionysiaques et mystères chrétiens ", RHR 223 [2006], p. 267 et 270-271).

120. R. TURCAN, «Dionysos dimorphos: une illustration de la théologie de Bacchus dans l'art funéraire ", Mélanges d'archéologie et d'histoire 70 (1958), p. 243-294 (particulièrement p. 251-253, 277-280).

121. Clément d'Alexandrie, Protreptique II, $22,4$.

122. On ne dispose que de peu de renseignements sur ces Bassarai : voir DILLon, o.c. (n. 35), p. 147.

123. Voir S.I. JOHNSTON et Fr. GRAF, Ritual Texts for the Afterlife. Orpheus and the Bacchic Gold Tablets, London/New York, Routledge, $2013^{2}$ [2007], p. 73-76, 90, 140, 149, 150-157.

124. Euripide, Bacchantes, 4 et 53. 


\section{RÉSUMÉS}

Cet article comporte deux parties liées entre elles : l'une étudie le «manger cru » dans le régime alimentaire des Grecs, l'autre le rituel ômophagique dionysiaque. De l'étude conjointe des sources littéraires et épigraphiques, il apparaît que ce dernier pourrait consister en une séquence de gestes révélant l'ambiguïté du dieu, entre animalité, humanité et divinité, et s'accordant avec la pratique alimentaire des Grecs.

This paper consists of two inter-related sections: the first addresses the consumption of "raw food" in the ancient Greek diet; the second deals with the Dionysian ritual of ômophagia. Literary and epigraphic evidence shows that this ritual performance could be interpreted as a sequence of actions revealing the ambiguity of the god, between animality, humanity and divinity, and in accordance with the practice of raw food consumption among the Greeks.

\section{AUTEUR}

\section{CARINE VAN LIEFFERINGE}

Université Libre de Bruxelles

Avenue F.D. Roosevelt, 50

B - 1050 Bruxelles

cvlieffe@ulb.ac.be 